\title{
PICOT as a Dual Regulator of Cardiomyocyte Hypertrophy and Inotropy
}

Yoram Etzion ${ }^{1}$, Janet Ozer ${ }^{1}$, Yaron Ishay ${ }^{2}$, Gideon Sahar $^{2}$ and Noah Isakov ${ }^{3 *}$

${ }^{1}$ Department of Physiology and Cell Biology, Faculty of Health Sciences, Ben Gurion University of the Negev, Beer Sheva, Israel

${ }^{2}$ Department of Cardiothoracic Surgery, Soroka University Medical Center, Beer Sheva, Israel

${ }^{3}$ The Shraga Segal Department of Microbiology, Immunology and Genetics and the Cancer Research Center, Faculty of Health Sciences, Ben Gurion University of the Negev, Beer Sheva, Israel

Rec date: Feb 24, 2014, Acc date: Feb 26, 2014, Pub date: Feb 28, 2014

"Corresponding author: Noah Isakov, The Shraga Segal Department of Microbiology, Immunology and Genetics, Faculty of Health Sciences, Ben Gurion University of the Negev, P.O.B. 653, Beer Sheva 84105, Israel, Tel: 972-8-6477267; Fax: 972-8-6477626; E-mail: noah@bgu.ac.il

Copyright: $\odot 2014$ Etzion Y, et al. This is an open-access article distributed under the terms of the Creative Commons Attribution License, which permits unrestricted use, distribution, and reproduction in any medium, provided the original author and source are credited

\section{Editorial}

The contractile function of the normal adult heart is able to adapt rapidly in response to increased systemic demand for blood supply. Moreover, the heart undergoes a growth process, known as hypertrophy, in response to increased workload. This adaptive response is aimed to augment cardiac pump function and decrease ventricular wall tension in the face of an increased workload. However, depending on the type of workload stimulus, the heart can either go through a benign fully adaptive response, characterized by the growth of individual cardiomyocytes and enhanced vascular perfusion and metabolism (physiological hypertrophy) [1], or a maladaptive response that over time induces fibrosis, diminished cardiac performance, ventricular dilatation and an arrhythmogenic substrate (pathological cardiac hypertrophy) [2]. In the adult heart, physiological hypertrophy is typically seen in well-conditioned athletes in response to exercise and in the maternal heart during pregnancy. In contrast, pathological cardiac hypertrophy is typically triggered by abnormal stress that is tonic in nature, such as hypertension, myocardial infarction and valvular dysfunction [3,4]. Initially, the response to such stress also appears to be adaptive. However, it becomes maladaptive over time and eventually leads to heart failure and excessive risk for sudden cardiac death [2].

Mammalian cardiomyocytes are incapable of responding to an increased workload by cell proliferation and their main way to deal with sustained overload is by size increment and formation of additional sarcomeres. The cardiomyocyte hypertrophy is apparently mediated by a change in the balance between pro-hypertrophic and anti-hypertrophic factors, and the cell signaling pathways that are regulated by these factors. While a significant number of endogenous hypertrophic activators have been identified and characterized $[1,5,6]$, much less is known about the nature and the mode of action of endogenous negative regulators of cardiac hypertrophy [7]. Such negative regulators may be clinically significant as potential targets in new protocols for attenuation of pathological cardiac hypertrophypromoting processes. Recent studies by Park and colleagues focused on the role of PICOT in cardiomyocytes and provided ample support for PICOT-mediated anti-hypertrophic and inotropic activities.

PICOT (PKC-interacting cousin of thioredoxin; also termed glutaredoxin 3) was first identified in 2000, in a search for PKC $\theta$ binding proteins which function as regulators of PKC $\theta$ in $\mathrm{T}$ lymphocytes, and potentially affect PKC $\theta$-dependent activation signals downstream of the activated $\mathrm{T}$ cell antigen receptors [8]. In a search for potential novel regulators of cardiac hypertrophy, Jeong et al. used a PCR-based subtractive RNA profiling of differentially regulated genes in the cardiac tissue of sham operated healthy rats, versus rats with cardiac hypertrophy, induced by sustained pressure overload due to transaortic constriction [9]. Among the genes exhibiting altered expression in the hypertrophied heart, PICOT was significantly elevated and Northern blot analysis showed a $\sim 3$-fold increase in the level of PICOT mRNA in the hypertrophied heart compared to the sham-operated hearts. A similar increase in PICOT mRNA expression was observed in vitro in neonatal rat cardiomyocytes responding to hypertrophic agonists, such as endothelin-1, and phenylephrine [9], suggesting that PICOT is a hypertrophy-inducible factor in cardiomyocytes. Transgenic mice with cardiac-specific overexpression of PICOT were significantly more resistant to pressure overloadinduced cardiac hypertrophy. Furthermore, PICOT overexpression substantially enhanced the ventricular function and exhibited inotropic activity reflected by the PICOT-induced increased cardiomyocyte contractility [9].

Studies of the molecular mechanism by which PICOT regulates cardiomyocyte functions revealed that PICOT interacts, via its carboxy-terminus, with the muscle LIM protein (MLP), and colocalizes at the sarcomeric Z-disc [10,11]. A major role of MLP in cardiomyocytes is to anchor the $\mathrm{Ca} 2+$-dependent phosphatase, calcineurin $(\mathrm{CN})$ to the Z-disc, thereby activating the NFAT-regulated signaling pathway [12]. Competitive interaction of overexpressed PICOT with MLP, inhibited MLP-CN binding, thereby displacing the $\mathrm{CN}$ from the Z-disc, leading to downregulation of the NFATdependent signaling pathway. In accordance with these findings, a partial inhibition of NFAT targeted genes, which were induced by pressure overload, were inhibited in the cardiac tissue of PICOT overexpressing transgenic mice [12]. The latter studies suggested that PICOT-induced inhibition of cardiac hypertrophy was mediated by PICOT binding to MLP, inhibition of MLP-CN complex formation, and negative regulation of the MLP-CN-NFAT signaling pathway.

To further analyze the physiological role of PICOT in the heart, Cha and Kim [13] prepared a PIOCT-deficient mouse line, which was found to be embryonic lethal, indicating a critical role for PICOT during embryogenesis. The PICOT $+/$ - heterozygous mice which were viable and exhibited $\sim 50 \%$ of the normal level of the PICOT protein in the heart, exhibited an exacerbated cardiac hypertrophy, compared to wild-type mice, in response to pressure overload [13]. In agreement with the above-mentioned data, cardiomyocytes from PICOT $+/$ - mice exhibited reduced contractility and their CN-NFAT signaling was significantly enhanced in response to pressure overload.

In an attempt to study the mechanism underlying the positive inotropic effect of PICOT, and further to the initial observation of PICOT binding to PKC $\theta$ and PKC $\zeta$ in human T lymphocytes [8] Oh et al. [14] performed a pull-down assay using GST-PICOT, and found 
the association of PICOT with PKCל in a mouse heart extract [14]. Furthermore, in vitro studies revealed that purified PICOT inhibits the catalytic activity of PKCל. Based on results obtained in additional elaborative studies, the authors concluded that PICOT exerts its inotropic effect by direct inhibition of $\mathrm{PKC} \zeta$, which in turn, downregulates two independent pathways: (i) By inhibiting PKCa, it leads to elevation of sarco/endoplasmic reticulum Ca2+-ATPase (SERCA) 2a activity and increased phosphorylation of phospholamban (PLB), which increases Ca2+ movement. (ii) By inhibiting protein phosphatase $2 \mathrm{~A}$ (PP2A) activity it promotes the phosphorylation of troponin I and $\mathrm{T}$, which regulate cardiomyocyte contractility.

PICOT is a 335 amino-acid long highly conserved ubiquitous protein, which is found predominantly in the cytoplasm, but also in the nucleus of some cell types or under specific activation conditions [15]. It possesses a monothiol N-terminal thioredoxin-like homology domain, followed by two copies of monothiol glutaredoxin-like homology domains [16], and is assumed to function as a protein redox regulators [17], although no evidence yet exists to substantiate this theory. More recent studies demonstrated a potential role for PICOT in iron homeostasis and hemoglobin maturation [18], cell growth regulation [19] and neoplastic processes [13,20], although further research is needed in order to clarify the exact biological functions of PICOT.

While the inhibitory role of PICOT in the induction of pathological cardiac hypertrophy in rodents has been established, it remains to be tested whether PICOT possesses similar roles in the human heart, and which of the human cardiac pathologies are associates with changes in PICOT expression and/or activity. Studies correlating clinical parameters with the expression of PICOT in cardiac tissue extracted during open heart surgery may pave a way to better understanding of the role played by this intriguing protein in the human heart. The use of PICOT as an efficient target for the prevention of cardiac hypertrophy awaits the discovery of PICOT-specific drugs that retain the normal physiological functions of cardiomyocytes under extreme stress conditions, without having detrimental effects on other tissues.

\section{Acknowledgment}

Work in our lab was supported in part by a BGU-FOHS Start-up Research Grant.

\section{References}

1. Maillet M, van Berlo JH, Molkentin JD (2013) Molecular basis of physiological heart growth: fundamental concepts and new players. Nat Rev Mol Cell Biol 14: 38-48.

2. van Berlo JH, Maillet M, Molkentin JD (2013) Signaling effectors underlying pathologic growth and remodeling of the heart. J Clin Invest 123: $37-45$.
3. Zanchetti A (2010) Hypertension: Cardiac hypertrophy as a target of antihypertensive therapy. Nat Rev Cardiol 7: 66-67.

4. Heineke J, Molkentin JD (2006) Regulation of cardiac hypertrophy by intracellular signalling pathways. Nat Rev Mol Cell Biol 7: 589-600.

5. Frey N, Katus HA, Olson EN, Hill JA (2004) Hypertrophy of the heart: a new therapeutic target? Circulation 109: 1580-1589.

6. Barry SP, Davidson SM, Townsend PA (2008) Molecular regulation of cardiac hypertrophy. Int J Biochem Cell Biol 40: 2023-2039.

7. Hardt SE, Sadoshima J (2004) Negative regulators of cardiac hypertrophy. Cardiovasc Res 63: 500-509.

8. Witte S, Villalba M, Bi K, Liu Y, Isakov N, et al. (2000) Inhibition of the c-Jun N-terminal kinase/AP-1 and NF-kappaB pathways by PICOT, a novel protein kinase $\mathrm{C}$-interacting protein with a thioredoxin homology domain. J Biol Chem 275: 1902-1909.

9. Jeong D, Cha H, Kim E, Kang M, Yang DK, et al. (2006) PICOT inhibits cardiac hypertrophy and enhances ventricular function and cardiomyocyte contractility. Circ Res 99: 307-314.

10. Jeong D, Kim JM, Cha H, Oh JG, Park J, et al. (2008) PICOT attenuates cardiac hypertrophy by disrupting calcineurin-NFAT signaling. Circ Res 102: 711-719.

11. Ohayon A, Dong G, Cohen S, Galperin M, Givoni A, et al. (2008) PICOT: A Trx- and Grx-like protein in a search of a function. Open Enzyme Inhibition Journal 1: 48-51.

12. Heineke J, Ruetten H, Willenbockel C, Gross SC, Naguib MS, et al. (2005) Attenuation of cardiac remodeling after myocardial infarction by muscle LIM protein-calcineurin signaling at the sarcomeric Z-disc. Proc Natl Acad Sci USA 102: 1655-1660.

13. Cha MK, Kim IH (2009) Preferential overexpression of glutaredoxin3 in human colon and lung carcinoma. Cancer Epidemiol 33: 281-287.

14. Oh JG, Jeong D, Cha H, Kim JM, Lifirsu E, et al. (2012) PICOT increases cardiac contractility by inhibiting PKCÎ activity. J Mol Cell Cardiol 53: 53-63.

15. Babichev Y, Isakov N (2001) Tyrosine phosphorylation of PICOT and its translocation to the nucleus in response of human $\mathrm{T}$ cells to oxidative stress. Adv Exp Med Biol 495: 41-45.

16. Isakov N, Witte S, Altman A (2000) PICOT-HD: a highly conserved protein domain that is often associated with thioredoxin and glutaredoxin modules. Trends Biochem Sci 25: 537-539.

17. Herrero E, de la Torre-Ruiz MA (2007) Monothiol glutaredoxins: a common domain for multiple functions. Cell Mol Life Sci 64: 1518-1530.

18. Haunhorst P, Hanschmann EM, Bräutigam L, Stehling O, Hoffmann B, et al. (2013) Crucial function of vertebrate glutaredoxin 3 (PICOT) in iron homeostasis and hemoglobin maturation. Mol Biol Cell 24: 1895-1903.

19. Cheng NH, Zhang W, Chen WQ, Jin J, Cui X, et al. (2011) A mammalian monothiol glutaredoxin, Grx3, is critical for cell cycle progression during embryogenesis. FEBS J 278: 2525-2539.

20. Ohayon A, Babichev Y, Pasvolsky R, Dong G, Sztarkier I, et al. (2010) Hodgkin's lymphoma cells exhibit high expression levels of the PICOT protein. J Immunotoxicol 7: 8-14. 